\title{
Photometry of some more neglected bright cataclysmic variables and candidates ${ }^{1}$
}

\author{
Albert Bruch \\ Laboratório Nacional de Astrofísica, Rua Estados Unidos, 154, \\ CEP 37504-364, Itajubá - MG, Brazil \\ (Published in: New Astronomy, Vol. 58, p. 53 - 60 (2018))
}

\begin{abstract}
As part of an effort to better characterize bright cataclysmic variables (CVs) and related systems which have received little attention in the past light curves of four systems (V504 Cen, KT Eri, Ret 1 and CTCV 2056-3014) are analyzed. For some of these stars no time resolved photometry has been published previously. While flickering is observed in all systems except Ret 1, it is particularly strong in V504 Cen and CTCV 2056-3014. In the latter star, a previously observed $15.4 \mathrm{~m}$ period, leading to its tentative classification as an intermediate polar, is probably spurious. Variations on time scales of hundredths of days observed in the pre-outburst light curve of the classical nova KT Eri continue after the outburst but appear not to be strictly periodic. Furthermore, the long term post-outburst light curve exhibits modulations with quasi-periods of quite different length. Thus, these variations cannot be due to aspect related variations in a system with a giant component similar to some recurrent novae. Instead, the system possibly exhibits variations with a period of $0.1952 \mathrm{~d}$ which may be orbital. However, any such conclusion still requires confirmation. The absence of flickering in Ret 1 indicates that the system probably does not contain an accretion disk. Instead, the observation of slow variations supports a previous suspicion of low amplitude variability with a period $>12 \mathrm{~h}$.
\end{abstract}

Keywords: Stars: novae, cataclysmic variables - Stars: individual: V504 Cen - Stars: individual: KT Eri-Stars: individual: Ret 1 = P831-57 - Stars: individual: CTCV 20563014

\section{Introduction}

Cataclysmic variables (CVs) are binary stars where a Roche-lobe filling late-type component (the secondary) transfers matter via an accretion disk to a white dwarf primary. It may be surprising that even after decades of intense studies of CVs there are still an appreciable number of known or suspected systems, bright enough to be easily observed with comparatively small telescopes, which have not been studied sufficiently for basic parameters to be known with certainty. In some cases even their very class membership still requires confirmation.

\footnotetext{
${ }^{1}$ Based on observations taken at the Observatório do Pico dos Dias / LNA
} 
Therefore, I started a small observing project aimed at a better understanding of these stars. Previous results have been published in a serie of papers by Bruch (2016, 2017a,b,c), Bruch \& Diaz (2017) and Bruch \& Monard (2017). Here, I present time resolved photometry of some more of these neglected systems. In most cases no such observations have been published before. I also retrieved data from publicly accessible archives which for some of the systems permit to draw valuable conclusions.

The main targets of this study are KT Eri, an old nova with cyclic brightness variations on the time scale of hundredth of days during quiescence, V504 Cen, a novalike variable which was recently identified to occasionally exhibit low states and thus belongs to the VY Scl subclass, and CTCV 2056-3014 which was classified as a candidate intermediate polar. Additionally, I briefly discuss a limited amount of observations of the CV candidate Ret 1 (= P831-57).

In Sect. 2 the observations and data reduction techniques are briefly presented. Sects. 3 - 6 then deal with the individual objects of this study. Finally, a short summary in Sect. 7 concludes this paper.

\section{Observations and data reductions}

All observations were obtained at the 0.6-m Zeiss and the 0.6-m Boller \& Chivens telescopes of the Observatório do Pico dos Dias (OPD), operated by the Laboratório Nacional de Astrofísica, Brazil. Time series imaging of the field around the target stars was performed using cameras of type Andor iKon-L936-B and iKon-L936-EX2 equipped with back illuminated, visually optimized CCDs. A summary of the observations is given in Table 1. Some light curves contain gaps caused by intermittent clouds or technical reasons. In order to resolve the expected rapid flickering variations typical for CVs the integration times were kept short. Together with the small readout times of the detectors this resulted in a time resolution of the order of $5 \mathrm{~s}$. In order to maximize the count rates in these short time intervals no filters were used. Therefore, it was not possible to calibrate the stellar magnitudes. Instead, the brightness is expressed as the magnitude difference between the target and a nearby comparison star, the constancy of which was verified through the observation of several check stars. A rough estimate of the effective wavelength of the white light band pass, assuming a mean atmospheric extinction curve, a flat transmission curve for the telescope, and a detector efficiency curve as provided by the manufacturer, yields $\lambda_{\text {eff }} \approx 5530 \AA$, very close to the effective wavelength of the Johnson $V$ band (5500 $\AA$; Allen 1973). Therefore, using $V$ magnitudes of the comparison stars as provided by Zacharias et al. (2013), it is possible to calculate approximate mean nightly visual magnitudes of the target stars. The reliability of these is attested by the excellent agreement with archival long-term light curves as demonstrated in the case of KT Eri in Fig. 1 (Sect. 3.1) where the yellow dot, which represents the magnitude of KT Eri during the observing nights discussed here, falls right on the long term visual light curve retrieved form the archives of the American Association of Variable Star Observers (AAVSO), Association Française des Observateurs d'Etoiles Variables (AFOEV), and British Astronomical Association, Variable Star Section (BAAVSS). The average nightly magnitude of the observed stars is included in Table 1. A list of the comparison stars for each target, taken from the UCAC4 catalogue (Zacharias et al. 2013), together with their $V$ magnitudes is given in Table 2.

Basic data reduction (biasing, flat-fielding) was performed using IRAF. For the construction of light curves aperture photometry routines implemented in the MIRA software system (Bruch 1993) were employed. The same system was used for all further data reductions and calculations. Throughout this paper time is expressed in UT. Timing analysis of the 
Table 1: Journal of observations

\begin{tabular}{llrrr}
\hline Name & Date & $\begin{array}{r}\text { Start } \\
(\mathrm{UT})\end{array}$ & $\begin{array}{r}\text { End } \\
(\mathrm{UT})\end{array}$ & $V$ \\
\hline V504 Cen & 2014 Apr 01 & $5: 17$ & $6: 59$ & 13.8 \\
& 2014 Apr 29 & $0: 03$ & $7: 17$ & 14.1 \\
& 2014 Apr 29/30 & $23: 15$ & $6: 33$ & 14.3 \\
KT Eri & 2014 Oct 23 & $2: 23$ & $6: 17$ & 14.8 \\
& 2014 Oct 24 & $5: 56$ & $7: 45$ & 14.8 \\
Ret 1 & 2016 Aug 09 & $4: 56$ & $8: 50$ & 14.2 \\
& 2016 Set 06 & $7: 24$ & $7: 31$ & 14.1 \\
& 2016 Set 07 & $4: 55$ & $5: 26$ & 14.1 \\
& 2016 Set 08 & $5: 08$ & $5: 40$ & 14.1 \\
CTCV & 2016 Set 09 & $5: 26$ & $8: 27$ & 14.1 \\
2056-3014 & 2015 Jun 09 & $7: 52$ & $8: 47$ & 17.2 \\
& 2015 Jun 10 & $5: 30$ & $8: 55$ & 17.4 \\
& 2015 Jun 11 & $6: 04$ & $8: 54$ & 17.6 \\
& 2016 Set 07/08 & $21: 58$ & $3: 38$ & 17.0 \\
& 2016 Set 08/09 & $21: 37$ & $2: 29$ & 17.1 \\
\hline
\end{tabular}

Table 2: Comparison stars

\begin{tabular}{lll}
\hline Target star & $\begin{array}{l}\text { Comparison star } \\
\text { (UCAC4) }\end{array}$ & $V$ \\
\hline V504 Cen & $249-063231$ & 11.995 \\
KT Eri & $400-006830$ & 12.869 \\
Ret 1 & $131-003250$ & 13.031 \\
CTCV 2056-3014 & $299-343578$ & 13.453 \\
\hline
\end{tabular}


data employing Fourier techniques was done using the Lomb-Scargle algorithm (Lomb 1976, Scargle 1982, Horne \& Baliunas 1986). The terms "power spectrum" and "Lomb-Scargle periodogram" are used synonymously for the resulting graphs.

\section{$3 \quad$ KT Eri}

KT Eri is a well-known classical nova detected in 2009 by Itagaki (2009). While the outburst has been extensively documented by many observers, the quiescent state has received less attention. In particular, photometry with high time resolution has never been published. This is the motivation to include the star in the present study.

Periodic variations at $0.09381 \mathrm{~d}(=135 \mathrm{~m}$ ) (or possible alias periods at $0.10348 \mathrm{~d}$ or $0.115412 \mathrm{~d}$ ) with an amplitude of 0.05 have been reported by C. Stockdale (Kato, vsnetalert $11755^{2}$ ). These are identified in the most recent on-line version of the Ritter \& Kolb catalogue (Ritter \& Kolb 2003) as the orbital period. However, in historical (pre-outburst) light curves Jurdana-Šepić et al. (2012) found a modulation of the brightness with a period of 737 days and a second one of 376 days, i.e., very close to half of the former. They interpret these variations as being due to a cool giant which is filling its Roche lobe, combined with a high orbital inclination. The presence of a cool giant is also supported by the small outburst amplitude of $\sim 9^{\mathrm{m}}$ which puts KT Eri in the vicinity of recurrent novae with evolved secondary stars.

Munari \& Dallaporte (2014) showed that the long-term modulations continue to be present after outburst with the same amplitude and phase (but note that their post-outburst observations cover only about one cycle of the 737 day period). Combining their observations with the pre-outburst data they derive a period of $752 \pm 2 \mathrm{~d}$, close to an alias of the $737 \mathrm{~d}$ period quoted by Jurdana-Šepić et al. (2012). However they are reluctent to accept this as the orbital period of KT Eri, bringing forward various arguments which contradict the scenario of a cataclysmic variable with an evolved secondary. Thus, the configuration of the system remains an open question.

\subsection{The long-term light curve}

Since the publication of Munari \& Dallaporte (2014) additional observations have become available. Fig. 1 (upper frame) contains the combined post-outburst light curve of KT Eri retrieved from the archives of the AAVSO, AFOEV and BAAVSS - noting that some of the data are common to more than one archive - binned into 1 day intervals. It contains two additional minima which are at least approximately in line with the expected minima of the supposed $752 \mathrm{~d}$ periodicity. These, together with the minimum already identified by Munari \& Dallaporte $(2014)^{3}$ are marked by back vertical marks in the figure.

I estimated the times of minima from Fig. 4 of Munari \& Dallaporte (2014). Adding the two new minima, a least squares fit yields periods of $732.6 \mathrm{~d}$ or $752.1 \mathrm{~d}$, depending on whether one assumes 38 or 37 cycles to have elapsed between the first minimum determined by Jurdana-Šepić et al. (2012) and the first post-outburst minimum. The predicted minimum epochs are marked as dashed red (shorter period) and green (longer period) vertical lines in Fig. 1. For the last two minima, they mark epochs considerably off the observed minimum epoch when the brightness of KT Eri was far from minimum. Folding the light curve on any of the two periods does not lead to a convincing phase dependent modulation. Moreover, an

\footnotetext{
${ }^{2}$ http://ooruri-kusastro-kyoto-u.ac.jp/mailarchive/vsnet-alter/11755

${ }^{3}$ The minimum close to JD 2456255 is not well defined in the archival light curves but is much better expressed in the data of Munari \& Dallaporte (2014) (see their Fig. 4).
} 

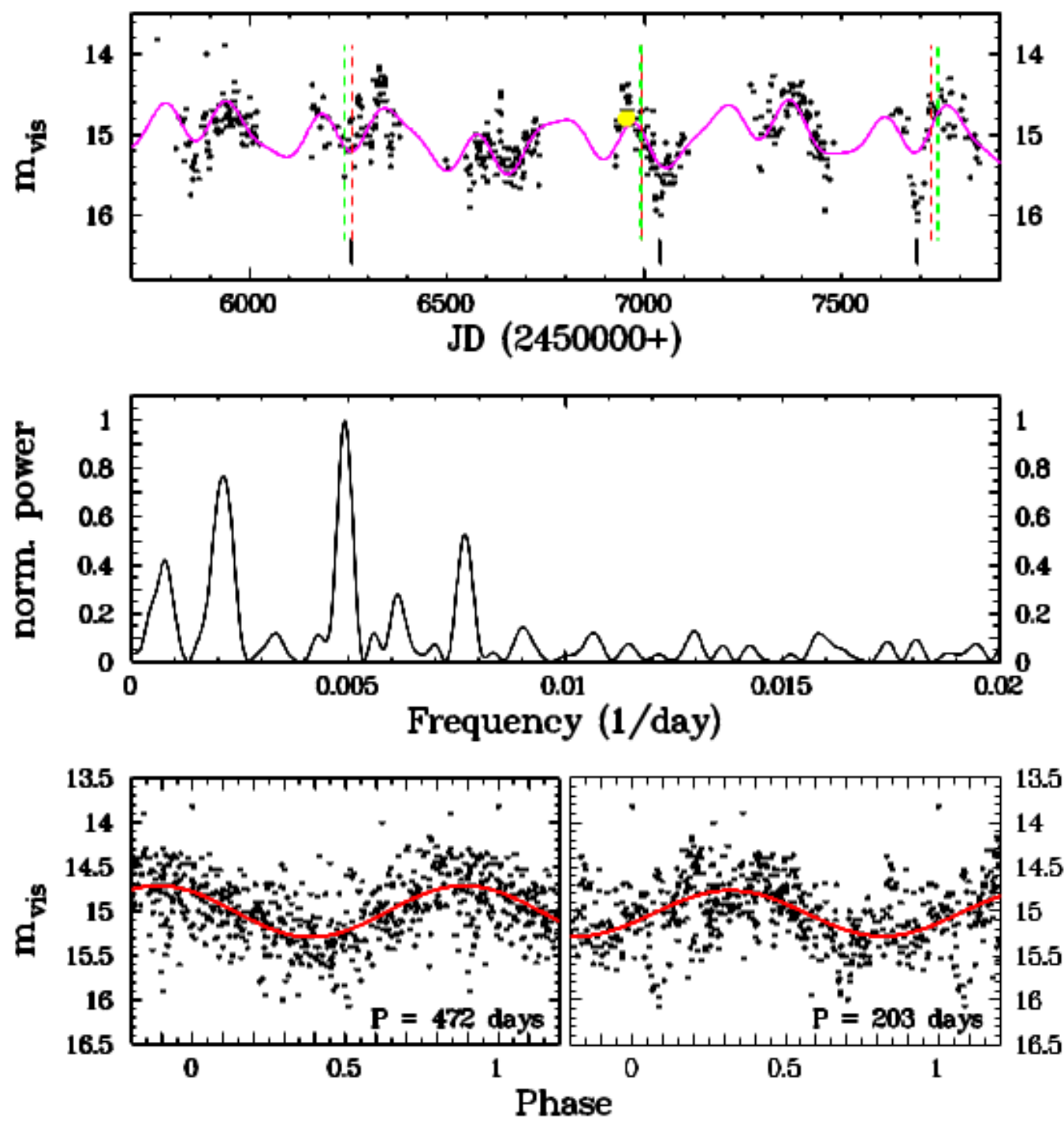

Figure 1: Upper frame: Post-outburst lightcurve of KT Eri (combined data from AAVSO, AFOEV and BAAVSS archives). The black tickmarks indicate epochs of supposed periodic minima. The broken red and green vertical lines indicate the predicted minimum epochs based on a linear least squares fit to the minimum epochs observed by Munari \& Dallaporte (2014) and the last two minima marked in the figure (for details, see text). The magenta curve is a four component least squares sine fit with periods fixed to the inverse of the frequencies of the four highest peaks in the Lomb-Scargle periodogram shown in the middle frame. The yellow dot marks the epoch and mean magnitude of the time resolved observations discussed in Sect. 3.2. Middle frame: Lomb-Scargle periodogram of the light curve in the upper frame. Lower frames: The light curve folded on the period 472 days (left) and 203 days (right), corresponding to the two highest peaks in the Lomb-Scargle periodogram. The phase zero point is arbitrary. The red curves represent least squares sine fits. 
additional apparently significant minimum in the light curve close to JD 2455860 does not fit at all into the picture of cyclic variations with one of the above periods. It may therefore be doubtful if one of them really represent a stable period of the system such as the orbital period should be.

Nevertheless, there are clear modulations in the post-outburst light curve. It was therefore subjected to a Fourier analysis. The Lomb-Scargle periodogram shown in the middle frame of Fig. 1 reveals that they cannot be described by a single period but rather indicates the simultaneous presence of several periods. The two most promient peaks occur at frequencies corresponding to $203 \mathrm{~d}$ and $472 \mathrm{~d}$. The light curve folded on these periods is shown in the lower part of the figure together with a least squares sine fit to the folded data (red curves). Note that the waveform is quite different from that shown in Fig. 4 of Jurdana-Šepić et al. (2012). For illustrative purposes I include in the upper frame of the figure a least square fit of the superposition of four sine curves with periods fixed to the inverse of the frequencies of the four highest peaks in the periodogram (magenta curve). While being far from perfect it is able to follow the general behaviour of the light curve.

I do not claim that any of the periods found in the post-outburst light curve or the periods reported by Jurdana-Šepić et al. (2012) or Munari \& Dallaporte (2014) are stable and persistent over long time scales. It appears premature to associate any of them to the orbital period of KT Eri. If the secondary star of the system is really a cool giant as advocated by Jurdana-Šepić et al. (2012), the observed time scales of the variations, their amplitude and their temporal behaviour are all compatible with those of semi-regular variables (in particular stars of type SRb) as defined by Kukarkin (1960). Orbital variation may also contribute to the variations in this scenario, but it would not be easy to separate them from intrinsic variations of the supposed giant secondary star.

\subsection{Time resolved photometry}

I obtained time resolved photometry of KT Eri in two subsequent nights in 2014, October. Expecting the white light magnitudes to be approximately equal to visual magnitudes, and noting that the average brightness was the same in both nights, leads to a location of these data as indicated by a yellow dot in Fig. 1.

The light curves, shown in Fig. 2, exhibit flickering on a modest magnitude scale and superposed upon longer time scale variations. Some basic flickering parameters were determined, adopting exactly the same procedures described in detail by Bruch (2017a). The results are summarized in Table 3. The flickering amplitude is parameterized by the FWHM of a Gaussian fit to the magnitude distribution of the individual data points in each light curve after subtraction of variations on time scales $>30 \mathrm{~m}$. It must be considered an upper limit since random noise broadens the distribution. The slope $\alpha_{\mathrm{ps}}$ of the linear part of the double logarithmic power spectrum, measured in the frequency range $-3<\log [f(H z)]<-2$ varies strongly between the two observing nights. This may be aggravated by the higher noise level in the second night (see Fig. 2). Consequently, the average of the scalegram parameter $\alpha_{\text {wav }}$ based on a wavelet analysis (Fritz \& Bruch 1998) has also a large statistical error. This is not so for the parameter $\Sigma$ which almost does not change. In agreement with the nature of KT Eri the measured values place the system well within the range occupied by novae in the $\alpha_{\text {wav }}-\Sigma$ plane (see Fig. 11 of Fritz \& Bruch 1998).

\subsection{The photometric period}

The photometric period of 0 d 09381 , quoted in the informal communication of Kato, not being well documented and confirmed, cannot be considered as the established orbital period of 


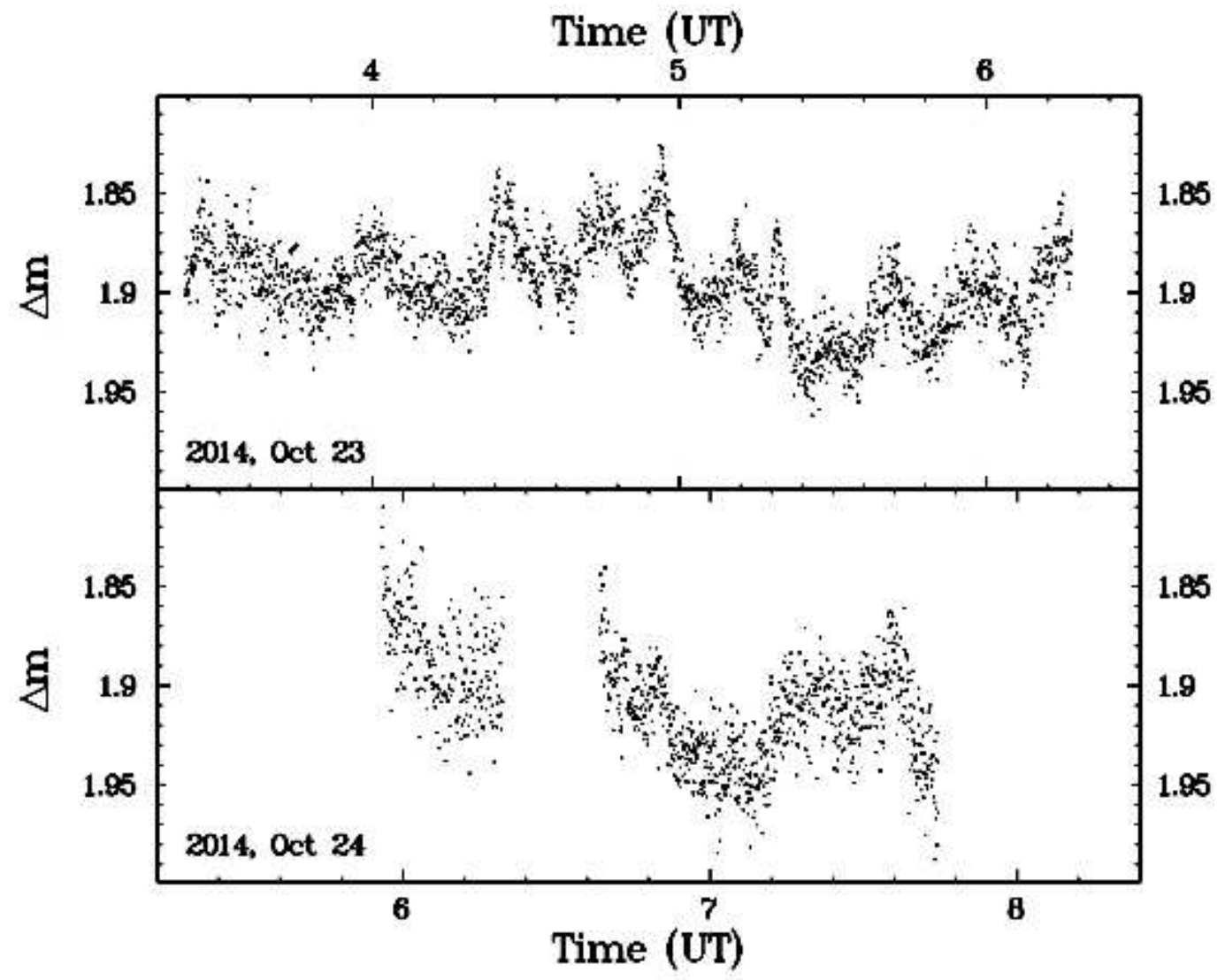

Figure 2: Light curves of KT Eri in two nights in 2014.

Table 3: Flickering parameters

\begin{tabular}{lccccc}
\hline $\begin{array}{l}\text { Object } \\
\text { Name }\end{array}$ & $\begin{array}{c}\text { Amplitude } \\
(\mathrm{FWHM})(\mathrm{mag})\end{array}$ & $\begin{array}{c}\alpha_{\mathrm{ps}} \\
\text { (power spectrum) }\end{array}$ & $\begin{array}{c}\alpha_{\text {wav }} \\
\text { (wavelet) }\end{array}$ & $\begin{array}{c}\Sigma \\
\text { (wavelet) }\end{array}$ & $\begin{array}{c}\text { Number of } \\
\text { light curves }\end{array}$ \\
\hline V504 Cen & $0.156 \pm 0.021$ & $-1.57 \pm 0.03(0.02)$ & $1.80 \pm 0.08$ & $-0.87 \pm 0.05$ & 3 \\
KT Eri & $0.038 \pm 0.008$ & $-1.67 \pm 0.38(0.27)$ & $1.54 \pm 0.45$ & $-2.43 \pm 0.02$ & 2 \\
CTCV 2056-3014 & $0.330 \pm 0.037$ & $-1.41 \pm 0.27(0.27)$ & $1.56 \pm 0.21$ & $-0.41 \pm 0.09$ & 5 \\
\hline
\end{tabular}


KT Eri. The corresponding observations must have been performed before 2009, December 30 (which is the date of vsnet-alert 11755) when the star had a visual magnitude brighter than $10^{\mathrm{m}}$ (Imamura \& Tanabe 2012), i.e. at least five magnitudes above the average post nova brightness. I may be doubtful that orbital variations manifest themselves in the light curve at this early stage after the nova outburst and at a light level so much above quiescence. The additional observations presented here are insufficient by far to be helpful in this context. However, the AAVSO data archive contains a series of light curves with a time resolution of (mostly) $2 \mathrm{~m}$, taken during 12 subsequent nights in 2013, March - April by F.-J. Hambsch, which are better suited for this purpose.

During the epoch of these observations KT Eri exhibited strong night to night variations. Therefore, the mean nightly magnitude was subtracted before the entire data set was subjected to a period analysis. A Lomb-Scargle periodogram of the results is shown in Fig. 3 (upper frame). It contains a family of peaks which are 1 /day aliases of each other. The strongest peak corresponds to a period of $0.1952 \pm 0.0013 \mathrm{~d}$ where the error was derived from the standard deviation of a Gaussian adjusted to the maximum. The data, folded on this period (with an arbitrary zero point for the phase), are plotted in the lower frame of the figure. Here, the red dots are the same data, binned in phase intervals of width 0.05 .

The amplitude of the modulation, determined from a least squared sine fit to the folded data is 0.058 , close to the value of 0.05 cited by Kato, while the period is very nearly twice as long as that reported by him. If one of these periods is really orbital in nature, Kato's value $2 \mathrm{~h} 15 \mathrm{~m}$ places KT Eri right at the lower edge of the orbital period gap of cataclysmic variables as defined by Knigge $(2006)^{4}$. The alternative period $(4 \mathrm{~h} 41 \mathrm{~m})$ placed the system close to the peak of the observed period distribution of novae derived from the catalogue of Ritter \& Kolb (2003).

However, it may be premature to identify any of these periods as orbital. Kato's value cannot be verified for lack or documentation. The light curves on which the present newly derived value is based are quite noisy, and so is the folded light curve. Moreover, old novae and novalike variables frequently exhibit (positive or negative) superhumps which are unstable over long time scales but in a limited data set may mimick orbital variations Examples include V603 Aql (Haefner \& Metz 1985, Bruch 1991, Patterson et al. 1993), TT Ari (Belova et al. 2013 and references therein; Smak 2013), KR Aur (Kozhevnikov 2007), V751 Cyg (Patterson et al. 2001, Papadaki et al. 2009), V795 Her (Papadaki et al. 2006), and V378 Peg (Kozhevnikov 2012). This issue as well as that of a possible giant nature of the secondary star (Sect. 3.1) could be settled by spectroscopic observations including time resolved radial velocity measurements which should be easily feasible considering the comparatively hight quiescent brightness of KT Eri.

\section{V504 Cen}

Originally, V504 Cen was thought be to a R Coronae Borealis star and it is listed as such in the General Catalogue of Variables Stars (Kholopov et al. 1984). Kilkenny \& Lloyd Evans (1989) rectify this classification and, based on the spectrum which shows strong and broad Balmer emission lines together with He I emisson, identify it as a cataclysmic variable. $U B V R I$ measurements at various epochs also reveal colours typical for CVs (see Bruch \& Engel 1994).

Kilkenny \& Lloyd Evans (1989) already suspected V504 Cen to be a VY Scl star, i.e. a CVs which occasionally exhibits periods of much reduced brightness. This classification

\footnotetext{
${ }^{4}$ Note that in a statistical analysis of the period distribution of classical novae Diaz \& Bruch (1997) could not verify the existence of the gap for this particular CV subtype.
} 

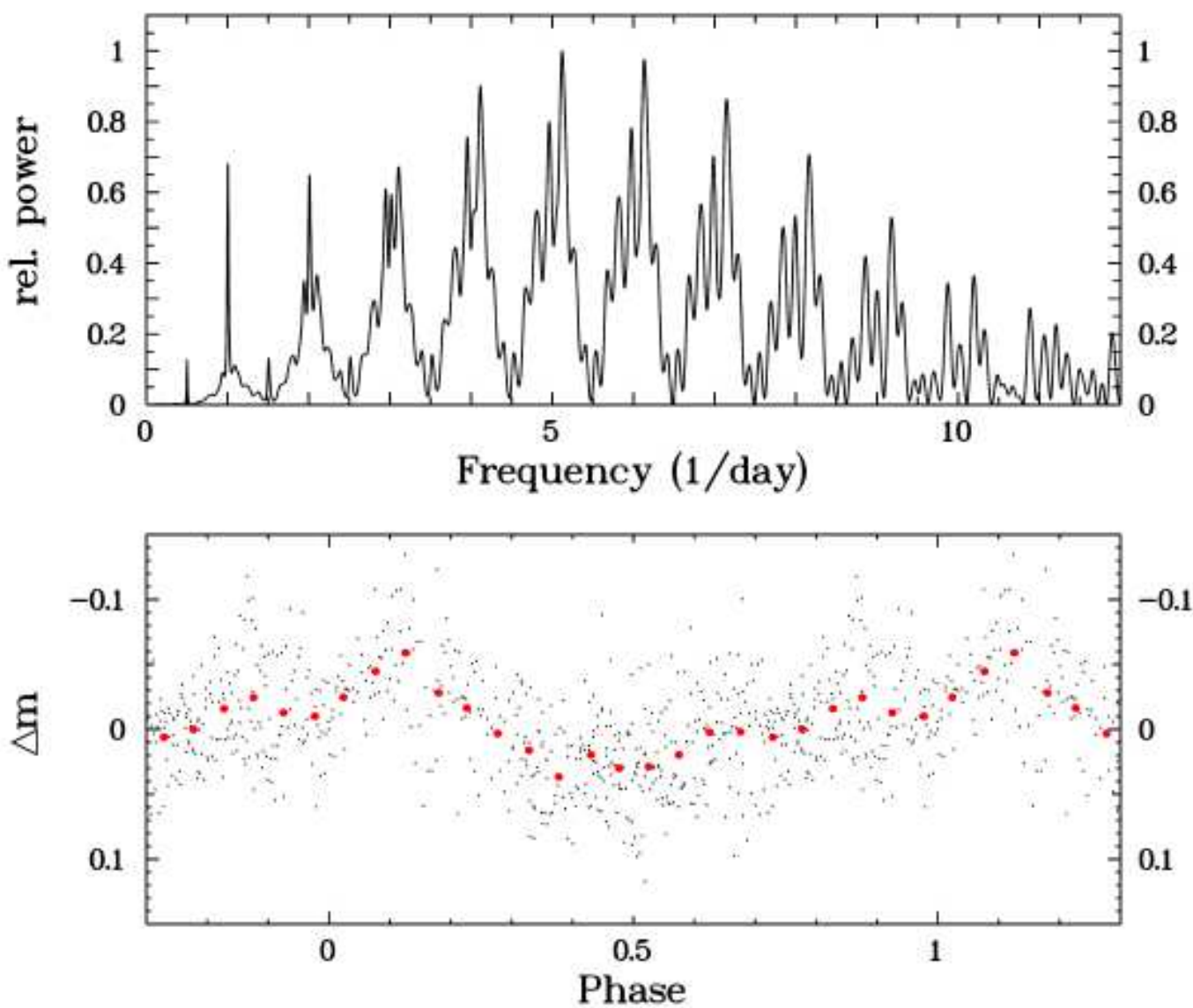

Figure 3: Top: Lomb-Scargle periodogram of time resolved light curves of KT Eri observed by F.-J. Hambsch in 2013. Bottom: Light curves folded on the period 0.1952 , corresponding to the highest peak in the power spectrum. The red dots represent the same data, binned into phase intervals of width 0.05 . The zero point of phase is arbitrary. 


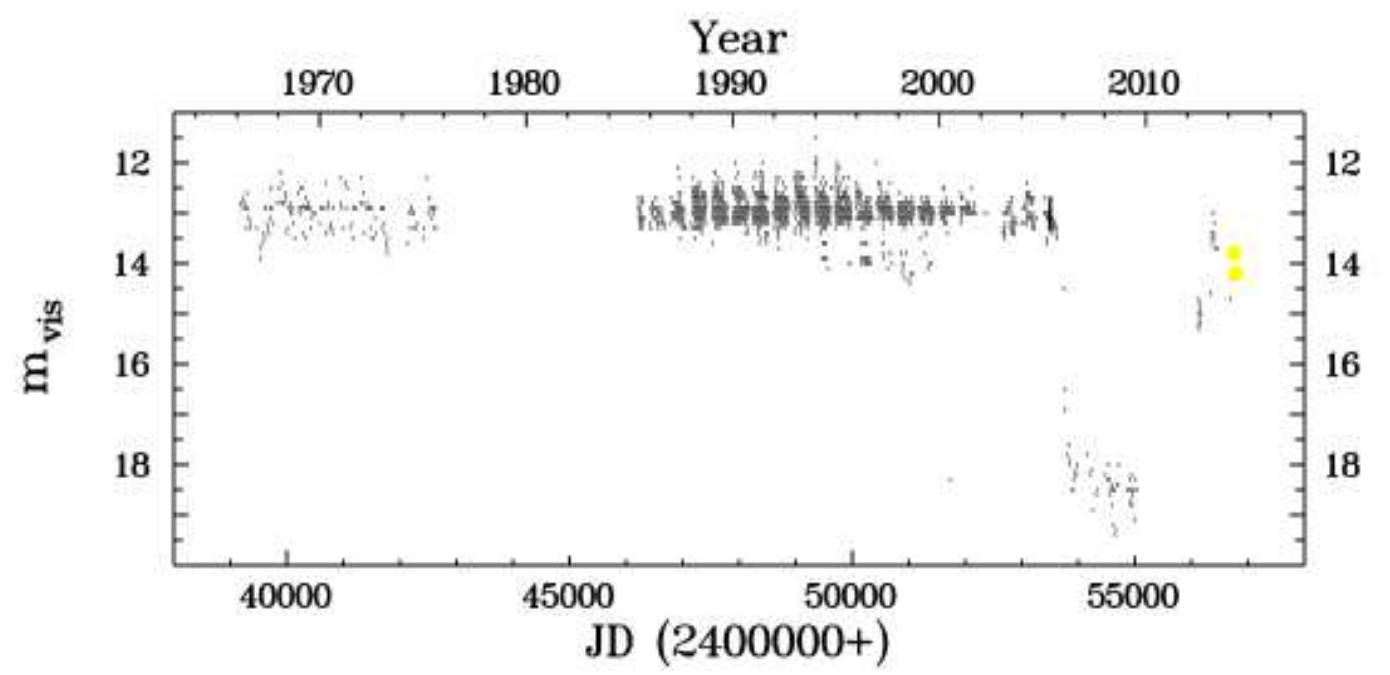

Figure 4: AAVSO long-term light curve of V504 Cen (one isolated data point at 8.2 on 2001, July 21, lies well above the upper boundary of the figure). The yellow dots indicate the epochs and average nightly magnitudes of the light curves listed in Table 1.

was confirmed by Kato \& Stubbings (2003) who, analyzing ASAS-3 data (Pojmanski 2002), found the star to be in a low state between March 2002 and January 2003. An extensive low state is also seen in the long-term AAVSO light curve (Fig. 4) between 2008 and 2010 (possibly until 2012), when V504 Cen hovered between $18^{\mathrm{m}}$ and $19^{\mathrm{m}}$.

Most of the knowledge available on V504 Cen comes from low state observations performed by Greiner et al. (2010). Long-term monitoring in the $I$-band, optical spectra and XMMNewton x-ray light curves all revealed an orbital period of $4.21 \mathrm{~h}$. The spectrum in the low state is dominated by narrow Balmer emission lines (emerging from broad absorption troughs in the higher Balmer lines) and numerous He I and low-ionization Fe lines also in emission.

No light curves with a high time resolution of V504 Cen, neither in the high nor in the low state, have been published. I obtained light curves in three nights in 2014 April which are shown in Fig. 5. The average nightly magnitude is indicated by yellow dots in Fig. 4 . The system was back from the low state but still about a magnitude below the long-term average high state brightness, or some tenths of a magnitude lower than the normal high state brightness reported by Kato \& Stubbings (2003), but compatible with the photometric measurements of Kilkenny \& Lloyd Evans (1989).

No variations on the orbital period are detectable, but V504 Cen exhibits spectacular flickering. The total amplitude reaches $0^{\mathrm{m}} \cdot 62$ on 2014 April $29^{5}$. While even higher amplitudes have occasionally been seen in some systems, this value places V504 Cen among the CVs with the strongest observed flickering (Beckemper 1995). Some basic flickering parameters of V504 Cen are included in Table 3.

There appears to be a tendency in the light curves for the appearance of comparatively isolated strong flares. This is particularly notable around $5 \mathrm{~h}$ UT on April 29. Similar events can also be seen at other times, albeit not being quite as obvious. To investigate this issue further, I have calculated power spectra of all light curves. They are shown in Fig. 6.

\footnotetext{
${ }^{5}$ The total range of variations on 2014 April 30 is even higher $(0.75)$, but this is due to longer term variation in this night which may not be related to flickering.
} 


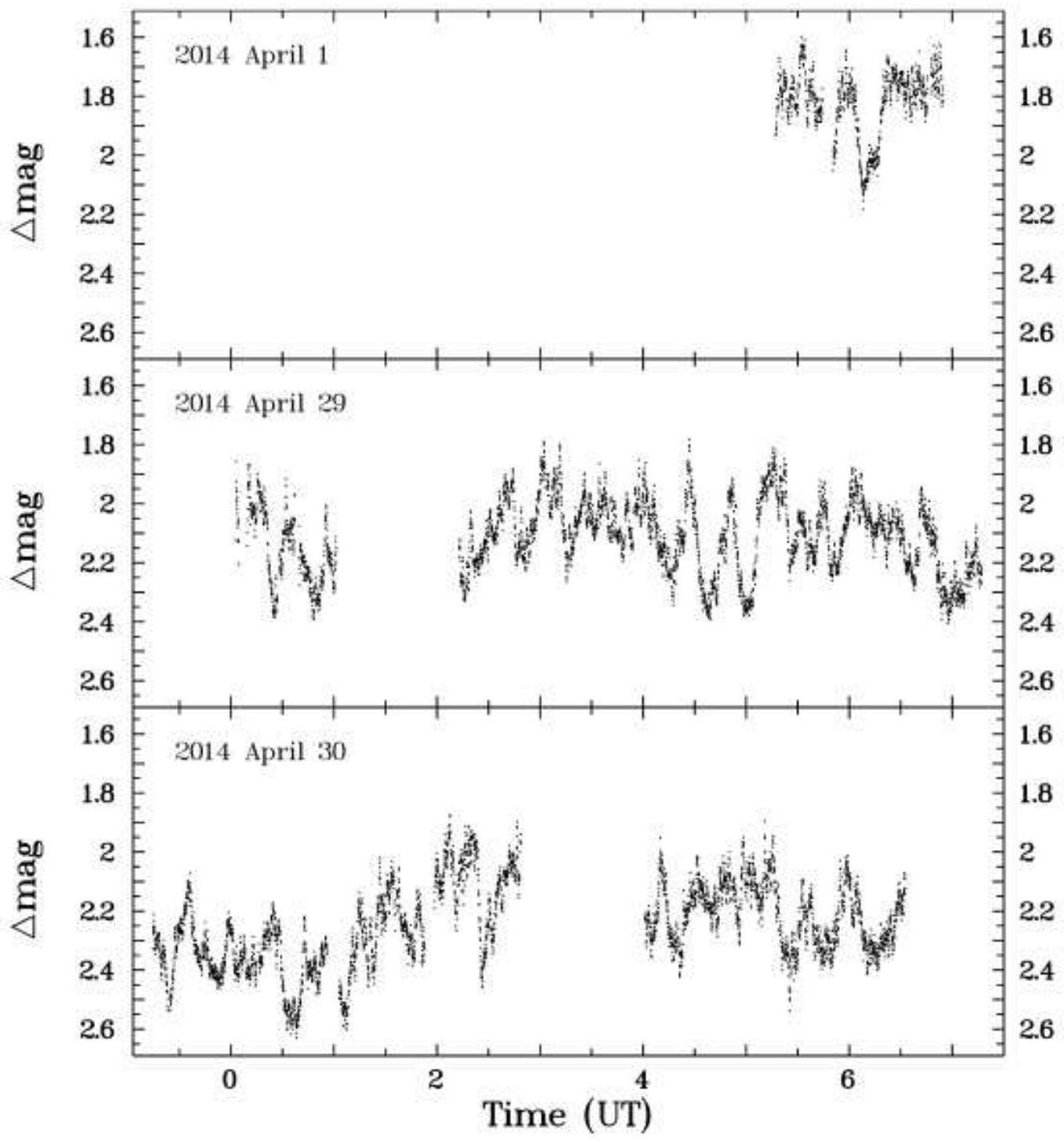

Figure 5: Time resolved white light photometry of V504 Cen during three nights in 2014 April. 
The light curve of April 30 exhibits clear long-term variations, being systematically fainter during the first two hours of observations. Since these will dominate the power spectrum at low frequencies, a smoothed version which follows only the slow trends and leaves the more rapid variations untouched was subtracted from the original light curve before calculating the power spectrum.

In all power spectra a conspicuous signal is seen at frequencies between 2 and 3 cycles per hour. While it is not resolved on April 1 because of the short duration of the light curves, it splits up into several components during the other nights. The frequencies of the individual peaks are not identical on April 29 and 30, indicating that the underlying signals are not strictly periodic. However, the clustering of power within a narrow frequency range in all nights can hardly be regarded as accidental. This suggests that the occurrence of the dominating flickering flares is not altogether stochastic but governed by a (quite imprecise) clock.

\section{$5 \quad$ CTCV 2056-3014}

Most of the knowledge about this star comes from Augusteijn et al. (2010) who present some spectroscopy and a limited amount of time resolved photometry. They derive an orbital period of $0.0732 \pm 0.0015 \mathrm{~d}$ from radial velocity variations. The spectrum is typical for a CV, showing the Balmer lines, He I and He II $\lambda 4686 \AA$ in emission. In a single light curve spanning $4.5 \mathrm{~h}$ the authors find indications for a period of $15.4 \mathrm{~m}$ which makes them suspect CTCV 2056-3014 to be an intermediate polar. This is supported by the detection of the star in x-rays (Haakonsen \& Rutledge 2009) and by the He II emission.

I observed CTCV 2056-3014 in three nights in 2015 and again in two nights in 2016 (see Table 1). The average magnitudes of the star range between $17^{\mathrm{m}} \cdot 2$ and $17^{\mathrm{m}} \cdot 6$ in 2015 . It was brighter in $2016\left(17^{\mathrm{m}} \cdot 0 \ldots 17^{\mathrm{m}} \cdot 2\right)$. Even so, all present observations show CTCV 2056-3014 to be fainter than found by Augusteijn et al. (2010): $16^{\mathrm{m}} \cdot 5$ (1996, Oct. 2), $15^{\mathrm{m}} \cdot 2$ (1996, Oct. 9) and $16^{\mathrm{m}} \cdot 4(2002$, July 7$)$.

Fig. 7 shows two light curves of the star, one from 2015 (upper frame) and the other one taken during the brighter state in 2016 (lower frame). The noisiness in particular at the low magnitude limit of the data reflects the faintness of the object for the telescope and the chosen integration time. Even so quite strong flickering is obvious with peaks reaching an amplitudes of 0.8 as well as more gradual variations on longer time scales. The basic flickering parameters are summarized in Table 3. The scalegram parameter $\Sigma$ has one of the highest values measured in any cataclysmic variable so far. It places CTCV 2056-3014 close to the upper border of the range found for magnetic systems and quiescent dwarf novae [?].

The variations above typical flickering times scales seen in the light curves of CTCV 20563014 are all too long to be identified as orbital variations. In order to verify the presence of $15.4 \mathrm{~m}$ oscillations as reported by Augusteijn et al. (2010) power spectra were calculated after removing low-frequency variations from the light curves. In no case an indication of a modulation at the corresponding frequency (or a consistent periodic modulation at any other frequency) was detected.

This may raise doubts upon the reality of the period found by Augusteijn et al. (2010) and consequently on their conclusion that CTCV 2056-3014 may be an intermediate polar. To address this question I reconstructed the light curve observed by Augusteijn et al. (2010) from their Fig. 27 and compare it to the light curve observed on 2016, Sep. 8 (upper frames of Fig. 8). In the latter the time resolution has been reduced to that of the former by

binning data points. Power spectra of both light curves were calculated after removal of low frequency variations by subtracting a third order polynomial fit (central frame of the 


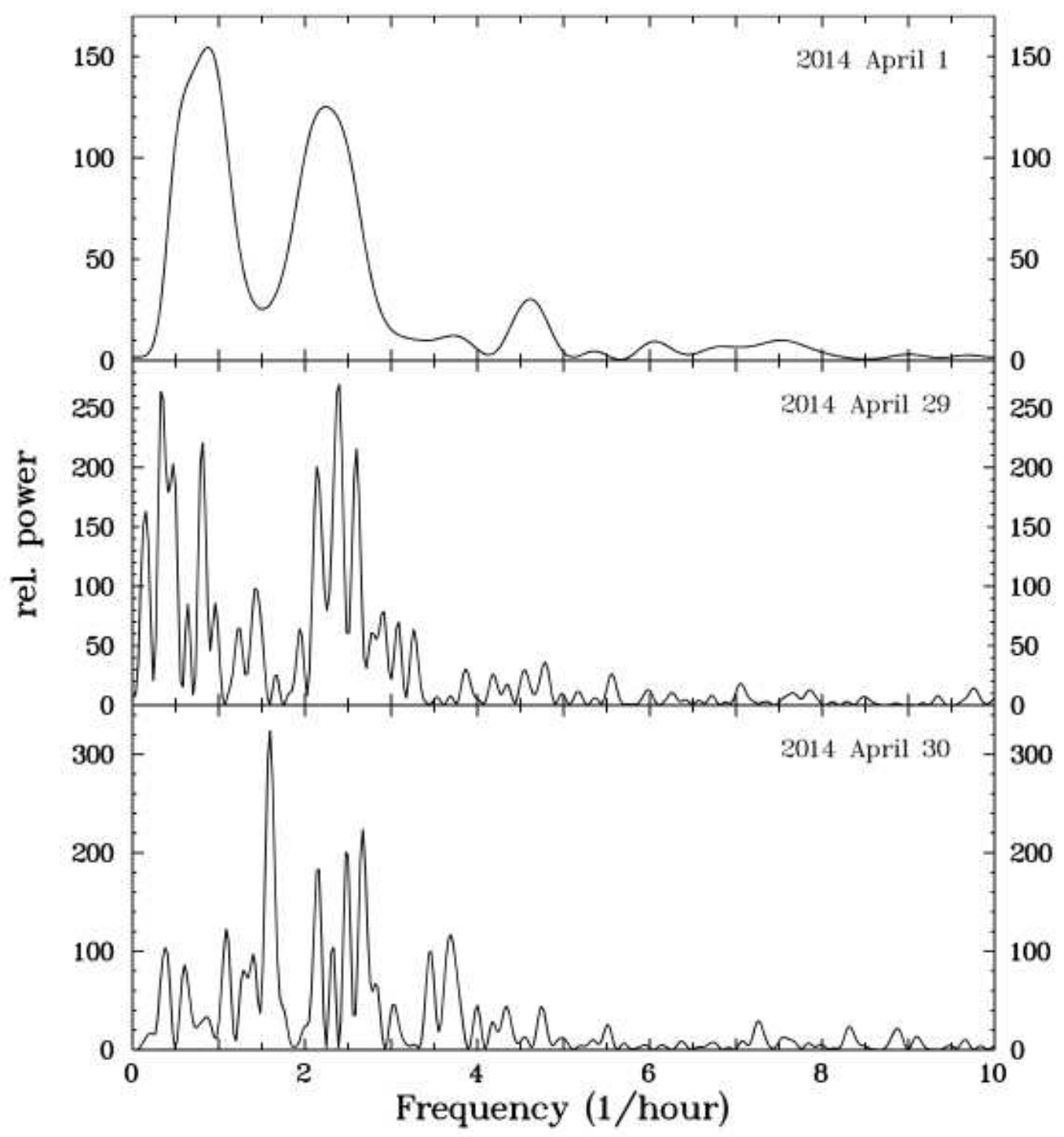

Figure 6: Power spectra of the light curves shown in Fig. 5. 


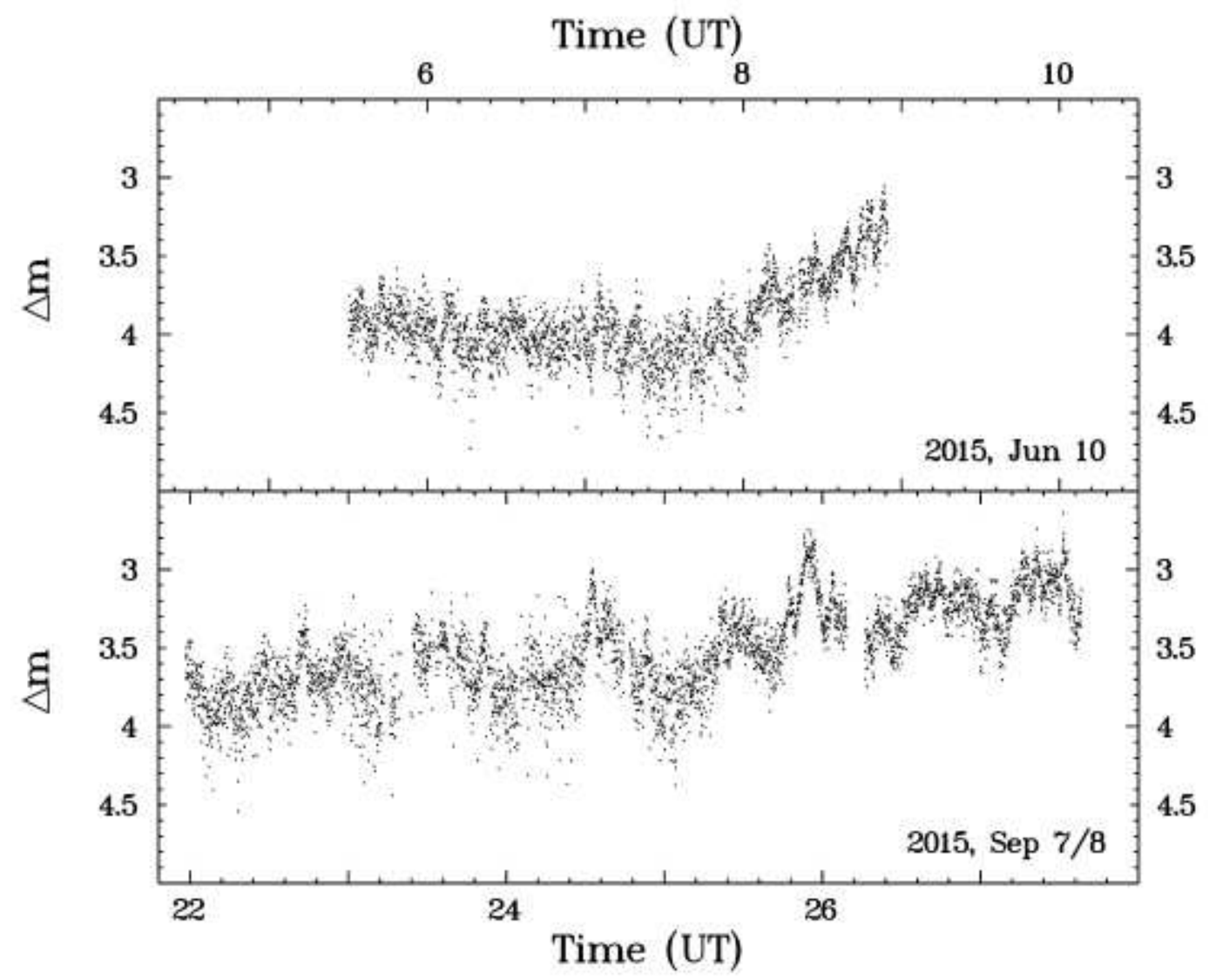

Figure 7: Light curves of CTCV 2056-3014 in two nights in 2015 and 2016. 
figure). The $15.4 \mathrm{~m}$ signal found by Augusteijn et al. (2010) (their Fig. 28) is reproduced (at a slightly different period of $15.5 \mathrm{~m}$ ). The power spectrum of the 2016 data shows no trace of this signal. Instead, there is a strong peak corresponding to $66.6 \mathrm{~m}$. The light curves (low frequency variation removed), folded on the respective periods, are shown as green dots in the lower frames of Fig. 8. The zero point of phase is arbitrary. The large black dots represent the same data, binned in phase intervals of width 0.1. The error bars are the standard deviations (black) of the data points in the respective phase intervals, while their red parts are the mean errors of the mean.

Regarding the black dots and the red error bars in the lower left frame of Fig. 8 it is seen that they are not unlike the graph shown in Fig. 29 of Augusteijn et al. (2010). However, the scatter of the original data points is much larger than the amplitude of the supposed periodic signal. The same is true in the lower right frame of the figure where a completely different period has been assumed. Instead of interpreting the variations as a real periodic signal, I strongly presume that they are caused by the accidental distribution of strong flickering flares in the light curve. This may serve as a warning that apparently periodic low amplitude variations in the presence of much stronger stochastic variations may well be spurious.

\section{$6 \quad$ Ret $1=$ P831-57}

Downes et al. (2005) quote Ret 1 as being of type nl: (i.e., novalike variable with uncertain classification). This is based on a communication by Rodgers \& Roberts (1994) who describe the spectrum of the star and presume it to arise from an accretion disk associated with a companion in orbit around a sub-dwarf $\mathrm{O}$ star. In later publications, Ret 1 is classified as a (candidate) pre-cataclysmic variable. Tappert et al. (2004) suspect a low amplitude $(<0.3)$ photometric variability with a period $>12 \mathrm{~h}$ which, however, could not be confirmed by Tappert et al. (2007). Godon et al. (2012) performed ultraviolet spectroscopy using the Far Ultraviolet Spectroscopic Explorer (FUSE). They concluded that the system does not have a disk and that the compact object (a white dwarf) is accreting from the wind of a secondary.

Since the nature of Ret 1 appears not to be established beyond any doubt, I observed some light curves of the star. Most of them are rather short and not helpful to reveal the nature of Ret $1^{6}$. Only one long continuous data set encompassing almost $4 \mathrm{~h}$ could be obtained. Unfortunately, these observations were seriously hampered by intermittent thin clouds which caused considerable scatter in the data. Therefore, the upper frame of Fig. 9 shows apart from the original differential magnitudes (data points deviating by more than 2.5 times the standard deviation have been removed) with respect to the comparison star also a smoothed version after applying a Gauss filter with a width $\sigma=0.2 \mathrm{~h}$ (red solid line). In spite of the noise it is obvious that no significant flickering is present, consistent with the notion that Ret 1 is not yet a cataclysmic variable. But there seems to be a smooth rise in magnitude of $\sim 0$. 05 over the observed time base. This is not seen in the differential light curve of a check star, which is shown in the lower frame of the figure. Another indication for a slight variability of Ret 1 comes from the statistics of the nightly average magnitudes: Their standard deviation is 0.0034 for the differential magnitudes between the target and the comparison star while it is only 0.0014 for the comparison star and three check stars. As a caveat it must be mentioned, however, that color dependent extinction together with

\footnotetext{
${ }^{6}$ Although Table 1 indicates a long time base for the observations of 2016, Sep. 09, the light curve really consists of two $\sim 1 \mathrm{~h}$ sections separated by a long gap caused by bad weather.
} 

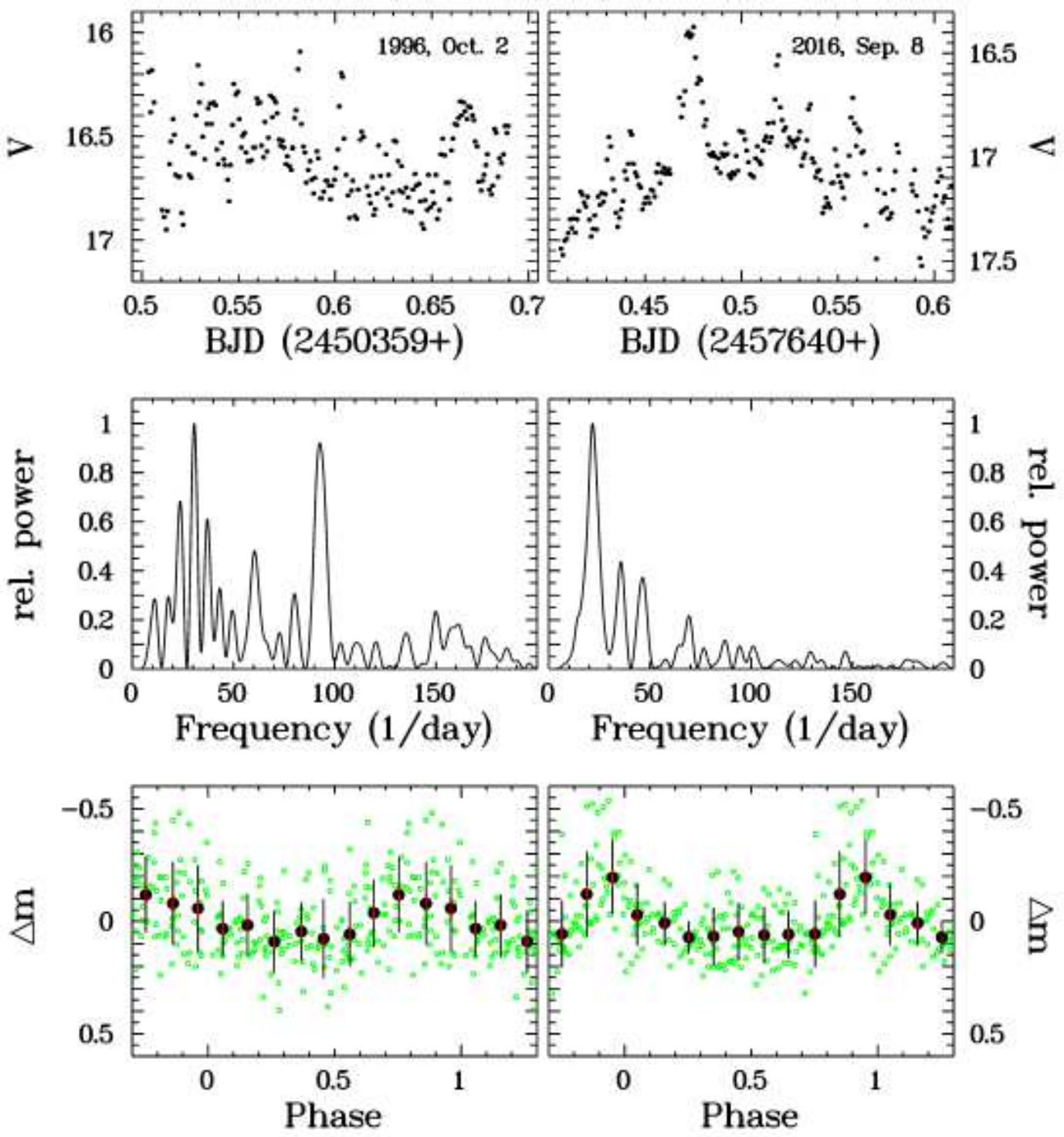

Figure 8: Upper frames: Light curve of CTCV-2056-3014 of 1996, Oct. 2, reconstructed from Fig. 27 of Augusteijn et al. (2010) (left), and light curve of 2016, Sep. 8, degraded to the same time resolution (right). Middle frames: Lomb-Scargle periodograms of the light curves after removal of low frequency variations by subtracting a third order polynomial fit. Lower frames: Light curves (green dots) folded on the periods corresponding to the dominant peaks in the periodograms [15.5 m (left) and $66.6 \mathrm{~m}$ (right)]. The black dots represent binned versions of the same data. The black (red) parts of the error bars are the standard deviations (mean errors of the mean) of the data within a given bin. 


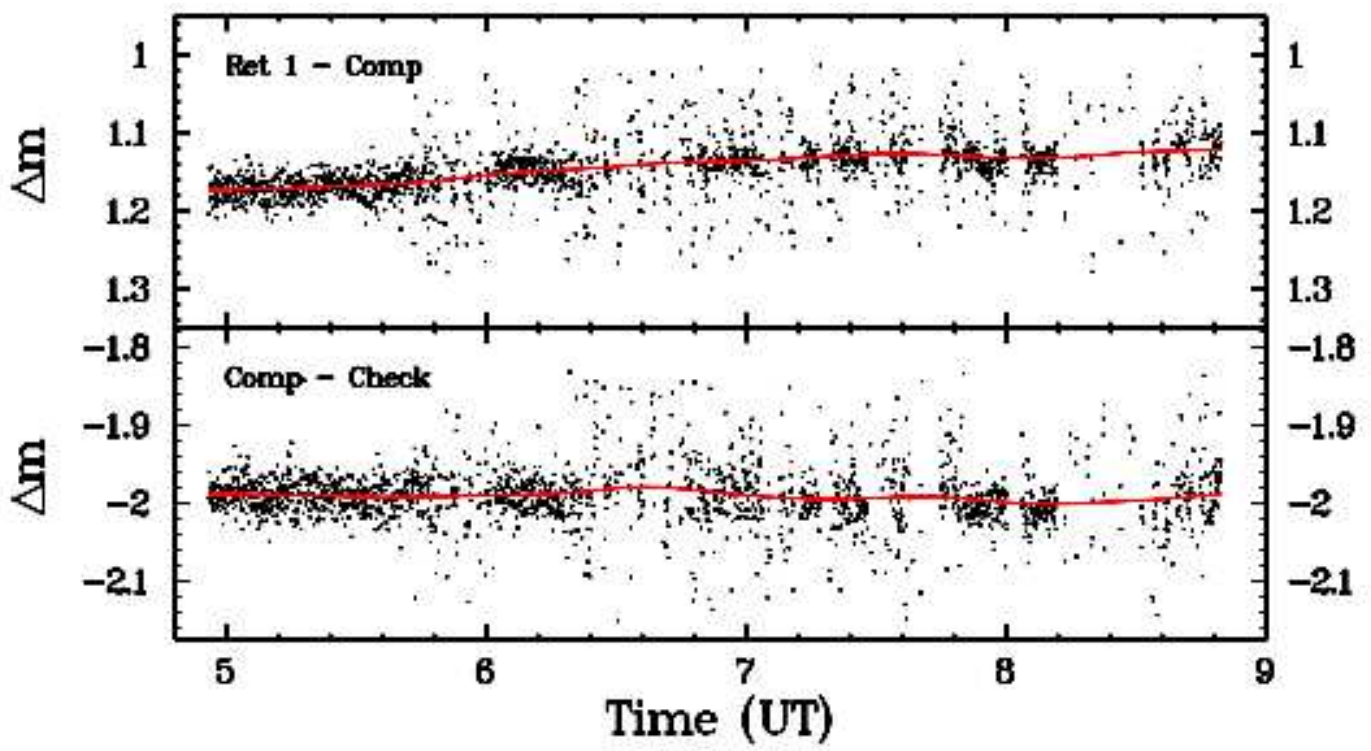

Figure 9: Differential light curve between Ret 1 and the comparison star UCAC4 131003250 (top) and between the comparison star and a check star (bottom). The red lines are smoothed versions of the original light curves.

different colours of target and comparison stars may cause variations of the differential magnitudes in these white light measurements.

\section{Summary}

I have presented time resolved photometry for an ensemble of four cataclysmic variables and candidates. For two of them (KT Eri and V504 Cen) no such observations have ever been published, while for the remaining systems only a quite limited amount of photometry has been performed in the past. The present observations are supplemented by data retrieved from publicly accessible data bases which provide valuable additional information.

The main findings of this study can be summarized as follows:

- Variations on time scales of hundredths of days observed in the classical nova KT Eri before its 2009 outburst (Jurdana-Šepić et al. 2012) continue after it returned to its quiescent state, but the period appears not to be quite stable. Moreover, the long term post-outbursts light curve contains modulations on different quasi-periods. None of the variations can confidently be interpreted as having a stable period. Thus, they cannot be explained as a geometrical effect in a system with an evolved stellar component in a configuration similar to some recurrent novae. In contrast, archival data contain indications of variations of KT Eri with a period of $0.1952 \mathrm{~d}$ (or an alias of this value) which may be orbital in nature. However, this finding still requires confirmation.

- V504 Cen was observed after an extended low state slightly below the long term high state magnitude. The limited amount of data does not permit to identify periodic variations in the light curve. However, the system exhibits spectacular flickering with indications that strong flares recur on a preferred time scale. Thus, they may not be entirely random events but governed by an underlying (quite imprecise) clock. 
- Just as V504 Cen, CTCV 2056-3014 flickers quite strongly. Previous claims of a 15.4 min period in its light curve (Augusteijn et al. 2010), interpreted as an indication for an intermediate polar nature of the system, could not be confirmed. Instead, it appears likely that apparently periodic low amplitude variations in the presence of much stronger stochastic variations (flickering) may well be spurious.

- Ret 1 was suspected to be a novalike variable [?]. More recent observations point instead at a nature as a pre-cataclysmic variable [?]. This is supported by the absence of flickering in the light curve. A possible rise of the light curve by 0.05 observed over the time interval of 4 hours as well as slight variations of the average magnitude in different nights may be due to orbital variations on longer time scales.

\section{Acknowledgements}

I gratefully acknowledge the use of the AAVSO, AFOEV, BAAVSS and ASAS data bases which provided valuable supportive information for this study.

\section{References}

Allen, C.W. 1973, Astrophysical Quantities, third edition (Athlone Press: London)

Augusteijn, T., Tappert, C., Dall, T., \& Maza, J. 2010, MNRAS, 405, 621

Beckemper, S. 1995, Statistische Untersuchungen zur Stärke des Flickering in kataklysmischen Veränderlichen, Diploma thesis, Münster

Belova, A.I., Suleimanov, V.F., Bikmaev, I.F., et al. 2013, Astron. Letters, 39, 111

Bruch, A. 1993, MIRA: A Reference Guide (Astron. Inst. Univ. Münster

Bruch, A. 1991, Acta Astron, 41, 101

Bruch, A. 2016, New Astr., 46, 90

Bruch, A. 2017a, New Astr., 52, 112

Bruch, A. 2017b, New Astr., 56, 69

Bruch, A. 2017c, New Astr., 57, 51

Bruch, A., \& Diaz, M.P. 2017, New Astr., 50, 109

Bruch, A., \& Engel, A. 1994, A\&AS, 104, 79

Bruch, A., \& Monard, B. 2017, New Astr., 55, 17

Diaz, M.P., \& Bruch, A. 1997, A\&A, 322, 807

Downes, R.A., Webbink, R.F., Shara, M.M., et al. 2005, J. Astron. Data, 11, 2

Fritz, T., \& Bruch, A. 1998, A\&A, 332, 586

Godon, P., Sion, E.M., Levay, K., et al. 2012, ApJ Suppl., 203, 29

Greiner, J., Schwarz, R., Tappert, C., et al. 2010, AN, 331, 227

Haakonsen, C.B., \& Rutledge, R.E. 2009, ApJ Suppl., 184, 138

Haefner, R., \& Metz, K. 1985, A\&A, 145, 311

Horne, J.H., \& Baliunas, S.L. 1986, ApJ, 302, 757

Imamura, K., \& Tanabe, K.. 2012, PASJ, 64, 120

Itagaki, K. 2009, CBET 2050, 1

Jurdana-Šepić, R., Ribeiro, V.A.R.M., Darnley, M.J, Munari, U, \& Bode, M.F. 2012, A\&A 537, A34

Kato, T., \& Stubbings, R. 2003, IBVS 5426 
Kholopov, P.N., Samus, N.N., Frolov, M.S., et al. 1984, General Catalogue of Variable Stars, 45th edition, Moscow

Kilkenny, D., \& Lloyd Evans, T. 1989, The Observatory, 109, 85

Knigge, C. 2006, MNRAS, 373, 484

Kozhevnikov, V.P. 2007, MNRAS, 378, 955

Kozhevnikov, V.P. 2012, New Astron., 17, 38

Kukarkin, B.V. 1960, IAU Transactions, 10, 398

Lomb, N.R. 1976, Ap\&SS, 39, 447

Munari, U., \& Dallaporte, S. 2014, New Astr., 27, 25

Papadaki, C., Boffin, H.M.J., Stanishev, V., et al. 2009, J. Astron. Data, 15, 1

Papadaki, C., Boffin, H.M.J., Sterken C., et al. 2006, A\&A, 456, 599

Patterson, J., Thomas, G., Skillman, D.R., Diaz, M. 1993, ApJ Suppl., 86, 235

Patterson, J., Thorstensen J.R., Fried, R., et al. 2001, PASP, 113, 72

Pojmanski, G. 2002, Acta Astron, 52, 397

Ritter, H., \& Kolb, U. 2003, A\&A, 404, 301

Rodgers, A.W., \& Roberts, W.H. 1994, IAU Circ., 6043

Scargle, J.D. 1982, ApJ, 263, 853

Smak, J. 2013, Acta Astron., 17, 453

Tappert, C., Gänsicke, B.T., \& Mennickent, R.E. 2004, Rev. Mex. AA, Conf. Ser., 20, 245

Tappert, C., Gänsicke, B.T., Schmidtobreick, L., Mennickent, R.E., \& Navarrete, F.P. 2007, A\&A, 475, 575

Zacharias, N., Finch, C.T., Girard, T.M., et al. 2013, AJ, 145, 44 